\title{
Regeneration of Dopaminergic Function in 6-Hydroxydopamine- Lesioned Rats by Neuroimmunophilin Ligand Treatment
}

\author{
Chunyi Zhang, ${ }^{1}$ Joseph P. Steiner, ${ }^{2}$ Gregory S. Hamilton, ${ }^{2}$ T. Phillip Hicks, ${ }^{1}$ and Michael O. Poulter ${ }^{1,3}$ \\ ${ }^{1}$ National Research Council of Canada, Institute for Biological Sciences, Ottawa, Ontario, Canada K1A OR6, ${ }^{2}$ Guilford \\ Pharmaceuticals, Baltimore, Maryland 21224, and ${ }^{3}$ Neuroscience Research Institute, Carleton University Ottawa, Ontario, \\ Canada K1S 5B6
}

Nonimmunosuppressant immunophilin ligands have been found previously to stimulate neurite growth in culture and to promote regeneration of peripheral and central nerve fibers in vivo. To further characterize the effectiveness of these ligands, we have investigated the effect of the immunophilin ligand GPI-1046 in 6-hydroxydopamine (6-OHDA)-lesioned rats. In unlesioned rats, tetanic stimulation of the white matter induced long-term potentiation (LTP) of corticostriatal synaptic transmission as indicated by a $40-100 \%$ increase in the field potential amplitudes recorded in striatal brain slices. Unilateral microinjection of 6-OHDA into the substantia nigra resulted in a loss of corticostriatal LTP and in significant abnormality of motor behavior as assessed by amphetamine-induced ipsilateral rotations. Daily treatment of 6-OHDA-lesioned rats with GPI-1046 (10 mg/kg, s.c.) for 1 week reduced amphetamineinduced rotations by $75 \%$ and greatly restored the striatal tyrosine hydroxylase immunostaining. In addition, GPI-1046 almost completely restored corticostriatal LTP in 6-OHDAlesioned animals. LTP in normal animals and that restored by GPI-1046 in lesioned animals were both blocked by the NMDA receptor antagonist APV, suggesting mediation by NMDA receptors. Both LTPs were sensitive to dopamine (DA) receptor antagonists. The nonselective DA receptor antagonist chlorpromazine and the selective D1-D5 receptor antagonist $\mathrm{SCH} 23390$ reduced the LTP by $90 \%$. These results demonstrate that the immunophilin ligand GPI-1046 can reverse the abnormalities in the substantia nigra-striatal dopaminergic system that are caused by 6-OHDA, thus providing a potential therapeutic agent for Parkinson's disease.

Key words: 6-OHDA; LTP; striatum; dopamine; substantia nigra; Parkinson's disease
Parkinson's disease (PD) has been a focus of intense study since it was first characterized more than a century ago. The pathophysiological basis of PD is the degeneration of the nigrostriatal dopaminergic system. Although replenishment of dopamine (DA) in the brain by administration of the DA precursor L-3,4dihydroxyphenylalanine has shown a certain usefulness in the treatment of PD, this treatment is at best palliative and does not address the underlying pathophysiology. A more desirable treatment would be the restoration of the dopaminergic system. To this end, cell-replacement therapy has been attempted with varying degrees of success. However, ethical concerns (using human fetal tissue) as well as cross-infection issues from xenotransplantation (from pig tissue) have limited this treatment strategy.

The search for PD treatments has received a boost from recent studies showing that immunophilin ligands such as GPI-1046 regenerate brain tissue in a number of neurodegenerative models (Steiner et al., 1997b). Immunophilins are a family of small protein molecules, of which FK506 binding proteins (FKBPs) and cyclophilins are members. They serve as cytosolic receptors for immunosuppressant drugs (e.g., FK506) and create a complex that binds to and inhibits the calcineurin phosphatase activity mediated by $\mathrm{Ca}^{2+}$-calmodulin. These events inhibit a cascade of transcriptional events preventing the expression of cytokines such as interleukin-2 in T-cells. However, it has become clear that

\footnotetext{
Received Nov. 20, 2000; revised April 4, 2001; accepted April 18, 2001.

Correspondence should be addressed to Dr. Michael O. Poulter, Neuroscience Research Institute, Carleton University, 1125 Colonel By Drive, Ottawa, Ontario, Canada K1S 5B6. E-mail: Michael_Poulter@carleton.ca.

Copyright (C) 2001 Society for Neuroscience 0270-6474/01/210001-06\$15.00/0
}

ligands for these cytosolic receptor protein families can be generated that are not immunosuppressive. In particular, selective ligands with no immunosuppressant activity were synthesized for the immunophilin FKBP12, which is highly expressed in the brain. Previous studies with GPI-1046 have shown that this nonimmunosuppressant ligand binds to F KBP12 and elicits increased neuritic sprouting. Both FK506 and nonimmunosuppressant FKBP immunophilin ligands enhanced the functional and morphological recovery of crushed sciatic nerve in rats (Steiner et al., 1997a). Of particular relevance to PD, immunophilin ligands such as GPI-1046 increase tyrosine hydroxylase (TH)-immunoreactive fibers and dopaminergic terminals in the striatum after selective lesions of the nigrostriatal dopaminergic system (Steiner et al., 1997b).

The fact that PD patients have considerable difficulty in initiating movements has led to the suggestion that the basal ganglia is involved in the "automatic execution of learned movements." In

This article is published in The Journal of Neuroscience, Rapid Communications Section, which publishes brief, peerreviewed papers online, not in print. Rapid Communications are posted online approximately one month earlier than they would appear if printed. They are listed in the Table of Contents of the next open issue of JNeurosci. Cite this article as: JNeurosci, 2001, 21:RC156 (1-6). The publication date is the date of posting online at www.jneurosci.org.

http://www.jneurosci.org/cgi/content/full/5443 
this regard, striatal long-term potentiation (LTP) (Centonze et al., 1999b) may be a neurophysiological correlate of this facilitated movement as well as addictive behaviors (Berke and Hyman, 2000). Thus, the idea that PD results in reduced synaptic plasticity through the loss of dopaminergic function has gained support (Arbuthnott et al., 2000). The aim of the present study was to assess whether the nonimmunosuppressant immunophilin ligand GPI-1046 influences the electrophysiological properties of striatal synaptic transmission, especially striatal LTP, after rats have been lesioned by microinjection of the substantia nigra with the neurotoxin 6-OHDA. Using this standard model of PD, our results show that these lesions of the substantia nigra area abolished striatal LTP, whereas subsequent (after establishment of the lesion) GPI-1046 treatment almost completely restores this activity.

\section{MATERIALS AND METHODS}

The PD animal model and treatment with GPI-1046. 6-OHDA was injected into the left substantia nigra to produce the PD model. Adult male Sprague Dawley rats weighing 200-225 gm were anesthetized with a combination of ketamine $(87 \mathrm{mg} / \mathrm{kg})$ and xylazine $(13 \mathrm{mg} / \mathrm{kg})$. The tip of a microinjection cannula was placed in the substantia nigra (coordinates relative to bregma: posterior, $-5.2 \mathrm{~mm}$; lateral, $2.0 \mathrm{~mm}$; and height, -8.0 $\mathrm{mm}$ ). 6-OHDA, dissolved in $0.4 \%$ ascorbic acid to prevent oxidation, was injected $(2 \mu \mathrm{g} / \mathrm{rat})$ through the cannula in a volume of $2 \mu \mathrm{l}$ within $3 \mathrm{~min}$. Control animals were injected with vehicle $(0.4 \%$ ascorbic acid) in the same manner. At 2-3 weeks after surgery, the rotational behavior induced by amphetamine ( $3 \mathrm{mg} / \mathrm{kg}$, i.p.) was assessed to evaluate the effectiveness of 6-OHDA injection. Rats showing strong ipsilateral rotation were regarded as successful subjects and divided into two groups. One group received injections of GPI-1046 (10 mg/kg, s.c., daily) for 1 week. GPI-1046 was dissolved in $100 \%$ ethanol and diluted with intralipid solution. The other (control) group received injections of vehicle only (intralipid and alcohol).

TH immunohistochemistry. Brain tissue was fixed with $4 \%$ paraformaldehyde overnight and transferred to $15 \%$ sucrose solution. Frozen slices of $40 \mu \mathrm{m}$ thickness were cut from brain areas containing the striatum and substantia nigra. Slices were washed three times with PBS containing $0.2 \%$ Triton X-100, followed by incubation with peroxide mix (ethanol/ $30 \% \mathrm{H}_{2} \mathrm{O}_{2} / \mathrm{PBS}$ at a ratio of $\left.1: 1: 8\right)$ for $30 \mathrm{~min}$. After washing, slices were blocked with universal blocking solution (Dako Diagnostics Canada, Mississauga, Canada) for $30 \mathrm{~min}$. Slices were then incubated with anti-TH antibody (1:2500 dilution; Pel-Freez Biologicals, Rogers, AR) at $4^{\circ} \mathrm{C}$ overnight, washed as described previously, and incubated with biotinylated secondary antibody (1:100 dilution) for $60 \mathrm{~min}$. After removal of secondary antibody, slices were incubated with freshly made avidinbiotin complex (Vector Laboratories, Burlingame, CA) for $30 \mathrm{~min}$, washed three times with PBS, and subsequently incubated with DAB solution (Vector Laboratories) for $10 \mathrm{~min}$. After washing with water, slices were transferred to glass slides for analysis.

Electrophysiological recordings from brain slices. The electrophysiological portion of the study was undertaken 2-3 weeks after treatment with vehicle or GPI-1046. Rats were anesthetized with $\mathrm{CO}_{2}$ and decapitated. Brains were removed and blocked in ice-cold sucrose artificial CSF (ACSF) bubbled with $95 \% \mathrm{O}_{2}$ and $5 \% \mathrm{CO}_{2}$. The sucrose ACSF had the following composition (in $\mathrm{mm}$ ): 248 sucrose, $5 \mathrm{KCl}, 1.2 \mathrm{NaH}_{2} \mathrm{PO}_{4}, 26$ $\mathrm{NaHCO}_{3}, 10$ D-glucose, $2.4 \mathrm{CaCl}_{2}$, and $1.3 \mathrm{MgSO}_{4}$. Brain slices of $300-400 \mu \mathrm{m}$ thickness containing the striatum were prepared with a vibratome. The slices were incubated for $1 \mathrm{hr}$ in gassed sucrose ACSF and then for another hour in normal ACSF. The normal ACSF had the same composition except that sucrose was replaced with $\mathrm{NaCl}(124 \mathrm{~mm})$, and $\mathrm{MgSO}_{4}$ was omitted to eliminate magnesium inhibition of NMDA receptors and therefore facilitate long-term potentiation. The use of sucrose ACSF was to prevent overexcitation of neurons during slice preparation.

A slice was placed in a recording chamber and continuously perfused with gassed ACSF warmed to $35^{\circ} \mathrm{C}$. The perfusion rate was adjusted to $2 \mathrm{ml} / \mathrm{min}$. Recording electrodes were glass micropipettes filled with ACSF having tip resistances of 4-6 M $\Omega$. Recordings were made from the striatum. Field potentials were evoked by stimulating the white matter with a concentric bipolar electrode with single pulses of $0.5 \mathrm{msec}$ delivered at $0.25 \mathrm{~Hz}$. Stimulation was triggered by computer. The stimulating intensity was adjusted to that yielding one-third to half maximal. Striatal LTP was induced by four high-frequency trains, each composed of 40 pulses at $100 \mathrm{~Hz}$, separated by intervals of 1-5 sec. This protocol was found in preliminary experiments to be more effective in inducing LTP than a long, continuous train. In the experiments in which the effects of the NMDA receptor antagonist APV or the DA receptor antagonists chlorpromazine and SCH23390 were tested on striatal LTP, administration of antagonists was started at least $30 \mathrm{~min}$ before LTP was induced. Data were collected, preamplified, displayed on an oscilloscope, and stored and analyzed with DataWave (Boulder, CO) software.

Drugs and chemical reagents. DL-2-amino-5-phosphonovaleric acid (DLAPV) was purchased from Precision Biochemicals (Vancouver, British Columbia, Canada). Chlorpromazine, SCH23390, and 6-OHDA were purchased from Sigma (St. Louis, MO). GPI-1046 was synthesized at Guilford Pharmaceuticals. Amphetamine was provided by Health Canada (Ottawa, Ontario, Canada).

Statistics. The amplitude of the field potential was measured from the zero line to the peak of the first, largest negativity. To measure LTP, control values were taken from the average of responses immediately before tetanic stimulation for a period of $10 \mathrm{~min}$ and were compared with the average of the responses recorded after the tetanus for a period of up to $100 \mathrm{~min}$. The numerical data reported in the paper are represented as mean \pm SEM. Comparisons between means were made with a $t$ test or an ANOVA test. A $p$ value of $<0.05$ was considered statistically significant.

\section{RESULTS \\ Effect of GPI-1046 on rotational behavior in 6-OHDA-lesioned rats}

Unilateral injection of 6-OHDA into the substantia nigra of rats creates a unilateral lesion and the characteristic consequence of stereotypical motor behavior (Ungerstedt, 1971b) in which rats will walk in circles (rotate) ipsilateral to the lesion when injected with amphetamine $(3 \mathrm{mg} / \mathrm{kg}$, i.p.). This rotational behavior develops gradually over days, reaching a plateau $\sim 3$ weeks after injection (Ungerstedt, 1971a). For this reason, we assessed the completeness of the lesions by testing for rotational behavior 2-3 weeks after 6-OHDA treatment. Most lesioned rats (72 of 80 rats) responded by rotating $\sim 15$ times per minute, whereas shamoperated rats showed no response. Those rats with no obvious motor deficits were excluded from the study.

Rats showing positive rotational behaviors were then divided into two groups, receiving either vehicle or GPI-1046 $(10 \mathrm{mg} / \mathrm{kg}$, s.c.) once per day. Treatment lasted 1 week, and the rotational tests were then repeated 1-2 weeks after the end of treatment. In vehicle-treated rats, the number of rotations appeared to be slightly greater than after the first trial (but statistically nonsignificant), whereas GPI-1046 treatment reduced the number of rotations observed over that seen during the first trial (78 \pm 13 vs $26 \pm 5 ; p<0.01$ ) (Fig. $1 A$ ). Vehicle- and GPI-1046-treated rats were then used for electrophysiological experiments (see below).

Brain tissue also was processed for tyrosine hydroxylase activity. 6-OHDA injection into the substantia nigra resulted in a large reduction of $\mathrm{TH}$ immunoreactivity not only in the substantia nigra but also in the striatum, indicating degeneration in the nigrostriatal DA neuronal system (Fig. 1B). Treatment of 6-OHDA-lesioned rats with GPI-1046 partially restored the striatal TH immunoreactivity (eight of eight rats tested). This was not accompanied by an increase in immunoreactivity in the substantia nigra, which was essentially identical between lesioned and treated animals. Thus, the increase in striatal TH immunoreactivity was not associated with regeneration or with the sparing of cell bodies in the substantia nigra. We also found that GPI-1046 was not cytoprotective. In one set of rats, GPI-1046 treatment was started immediately before 6-OHDA injection. The lesion size, degree of rotational behavior, and immunoreactivity were the 


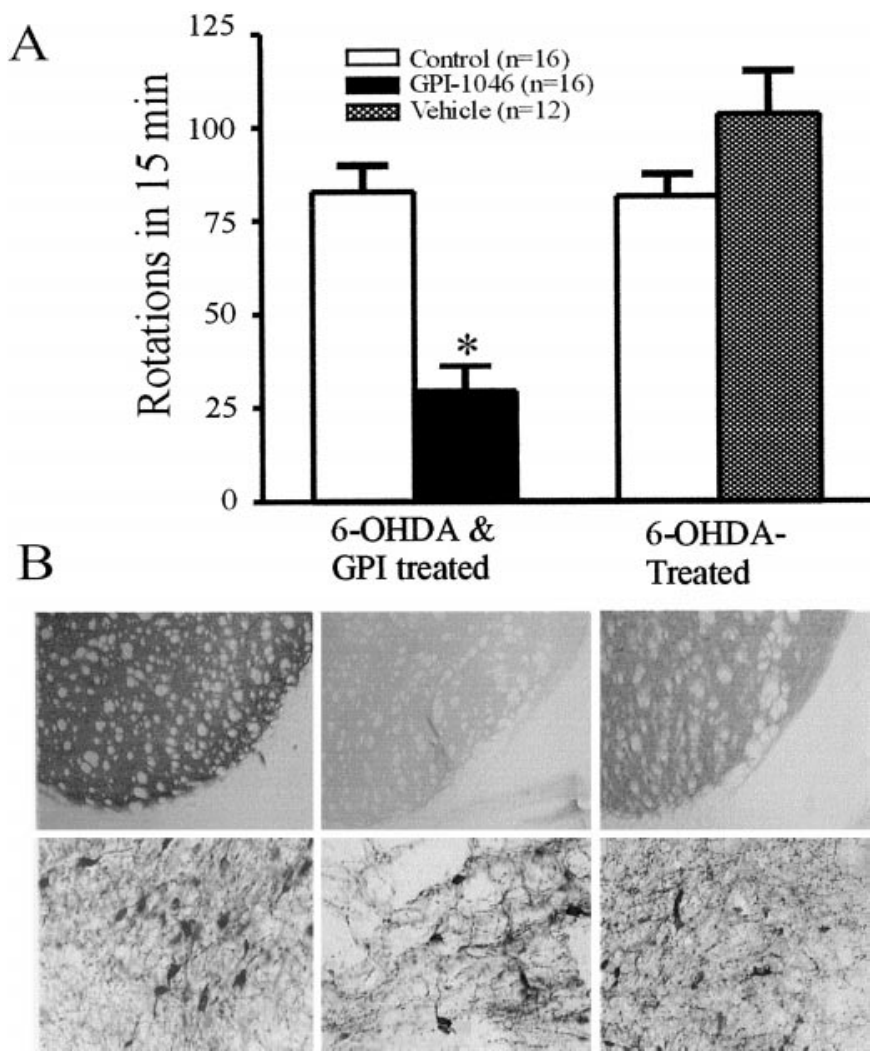

Figure 1. A, Summary of behavioral tests. 6-OHDA-treated rats were tested before and after GPI-1046 or vehicle treatment. In the GPI-treated group, the number of ipsilateral rotations was significantly decreased, whereas in the untreated group there was a trend toward an even larger amount of rotations. $B$, Tyrosine hydroxylase immunohistochemistry in the striatum (top row) and substantia nigra (bottom row). Left panels, Sham-operated substantia nigra. Middle panels, 6-OHDA injected into the substantia nigra. Right panels, 6-OHDA injected and subsequently treated with GPI-1046.

same as in the animals injected with 6-OHDA but not treated with GPI-1046 (data not shown). These data imply that the increased immunoreactivity in the striatum arose from resprouting of substantia nigra neurons that were spared from the lesion (estimated to be $\sim 5-10 \%$ ) or other dopaminergic nuclei (perhaps the lateral substantia nigra, which was primarily unaffected by the injections).

\section{Striatal LTP and its absence in 6-OHDA-lesioned rats}

To identify the electrophysiological correlates to the observed whole-animal behavior and the altered $\mathrm{TH}$ immunoreactivity, we prepared corticostriatal brain slices from normal, lesioned, and vehicle-treated rats, as well from as lesioned and GPI-1046treated rats. Extracellular recordings measuring the field potentials evoked by stimulation of the corticostriatal network were made. Stimulation of the cortical white matter induced a field potential similar to that observed by others and reminiscent of field potentials in the hippocampus. As reported previously in the absence of $\mathrm{Mg}^{2+}$ ion in the ACSF, high-frequency stimulation of the white matter of normal rats induces LTP (Calabresi et al., 1994, 1997; Centonze et al., 1999b). In unlesioned rats, tetanic stimulation produced an increase in the amplitude of the field potential of $64 \pm 8 \%(n=10 ; p<0.01)$ (Fig. $2 A$, sample and group data). However, this striatal LTP was essentially absent in 6-OHDA-lesioned rats (field potential amplitude increased by
$1.8 \pm 4 \% ; n=8 ; p>0.05)($ Fig. $2 A)$. These data indicate that the striatal LTP is abolished by damage to the nigrostriatal dopaminergic system.

Treatment with GPI-1046 of the 6-OHDA-lesioned rats that had exhibited extensive turning behavior indicated that their striatal LTP was restored by up to $85 \%$ compared with the level observed in sham-operated animals. The amplitude of the test field potential after high-frequency stimuli in GPI-1046-treated, lesioned rats was increased by $55.0 \pm 5.4 \%(n=21)$ (Fig. $2 B)$ of control amplitude, which was significantly greater than that seen in untreated animals (Fig. 2C). Maintenance of the restored LTP was the same compared with normal animals (Fig. $2 A, C$ ). In both GPI-1046-treated and normal rats, LTP was maintained up to 90 min after the stimulus. These data indicate a parallel recovery of neural plasticity and behavioral normality in the PD animal model.

\section{Properties of the regenerated striatal LTP}

We subsequently characterized what controls striatal LTP to determine the synaptic mechanisms regenerated in the GPI-1046treated animals. First, we tested whether, as in the hippocampus, striatal LTP was mediated by NMDA receptors. In the presence of the NMDA receptor antagonist APV $(50 \mu \mathrm{M})$, tetanic stimulation produced statistically nonsignificant increases in the amplitude of the field potentials in both sham-operated rats and in rats that were lesioned with 6-OHDA and treated with GPI-1046 $(3.7 \pm 3 \% ; n=5)$ (Fig. $3 A-C)$. Thus, in both treatment groups LTP induction was dependent on NMDA receptor-mediated synaptic transmission.

In addition, we determined that the striatal LTP requires an intact nigrostriatal dopaminergic system because DA receptor antagonists blocked striatal LTP. The nonselective antagonist chlorpromazine blocked LTP induction (test field potential was increased by only $2.6 \pm 3 \% ; n=5$ ) (Fig. 3). Second, the selective D1-D5 receptor antagonist SCH23390 prevented striatal LTP (the field potential was only $6.0 \pm 3 \%$ bigger than control; $n=5$ ) (Fig. 3). Therefore, these data indicated that GPI-1046 restores corticostriatal LTP by regenerating dopaminergic function in the striatum.

\section{DISCUSSION}

We have correlated regenerated TH immunoreactivity and have reduced the stereotypical turning behavior produced by GPI-1046 with the regeneration of corticostriatal LTP. Thus, the loss of DA terminals in the striatum (as indicated by the loss of TH immunoreactivity) is accompanied by the loss of LTP induced by high-frequency stimulation of the corticostriatal pathway. When the striatal immunoreactivity was partially restored by GPI-1046, striatal LTP was similarly restored. In addition, the damage was largely reversed by a single course of drug treatment.

However, there was no reduction in cell death in the substantia nigra. This implies that the increased striatal $\mathrm{TH}$ immunoreactivity results from increased sprouting from cells originally spared from neurotoxic insult in the substantia nigra or alternatively from other DA-containing nuclei. This enhanced sprouting would be consistent with previous results showing that GPI-1046 increased neurite growth from PC12 and dorsal root ganglia cells in culture (Steiner et al., 1997a). The mechanism of how neurophilin ligands induce enhanced sprouting is not well understood at this time. GPI-1046 binds to the phosphatase FKBP12, ultimately leading to a reversal of the neural deficits (Steiner et al., 1997a). 
Figure 2. $A$, Striatal LTP in normal rats and its absence in 6-OHDA-lesioned rats. $B$, The restoration (by GPI-1046) of striatal LTP in 6-OHDA-lesioned rats. $C$, Summary of the LTP data. $D$, Illustrations showing normal striatal LTP $(a)$, its absence in 6-OHDA-lesioned rats $(b)$, and its recovery after treatment with GPI-1046 (c).
Figure 3. Striatal LTP and its antagonism. $A$, LTP in GPI1046-treated animals. $B$, Blockade of the LTP by $50 \mu \mathrm{M}$ APV. $C$, Summary of the antagonism of LTP by APV. $D$, Antagonism of striatal LTP by the nonselective DA receptor antagonist chlorpromazine $(10 \mu \mathrm{M})$. E, Antagonism of striatal LTP by the selective D1-D5 receptor antagonist SCH23390 (5 $\mu \mathrm{M})$. Note the short-term depression in the presence of APV and chlorpromazine. $F$, Summary of the antagonism of striatal LTP by dopamine receptor antagonists.
A
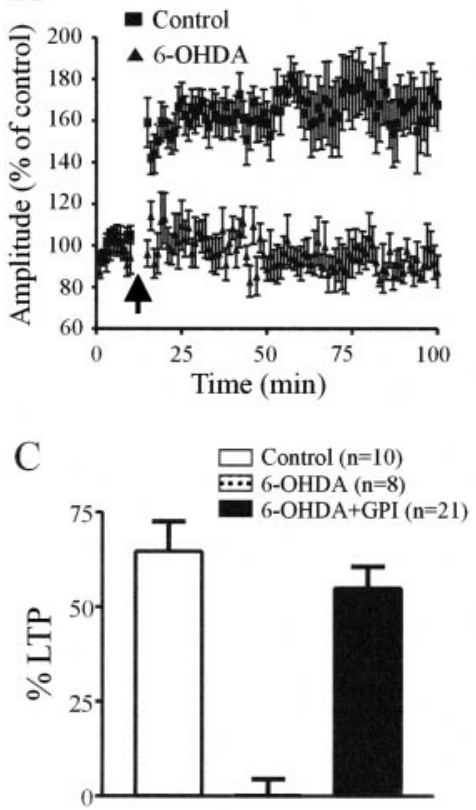

A

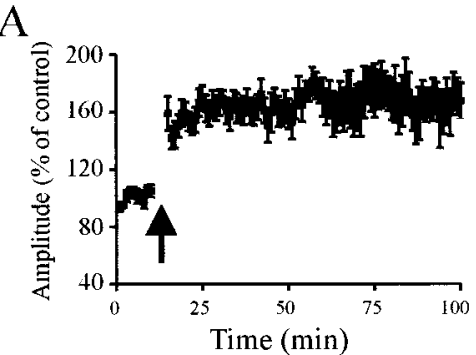

$\mathrm{B}$

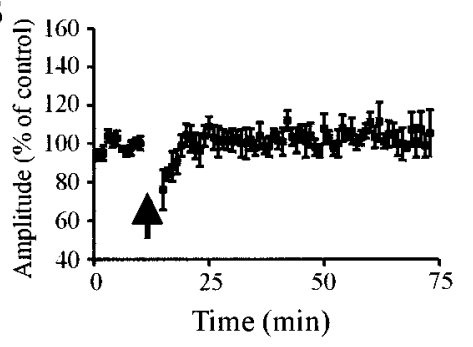

C

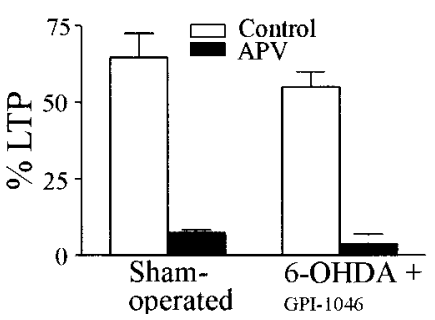

$\mathrm{B}$

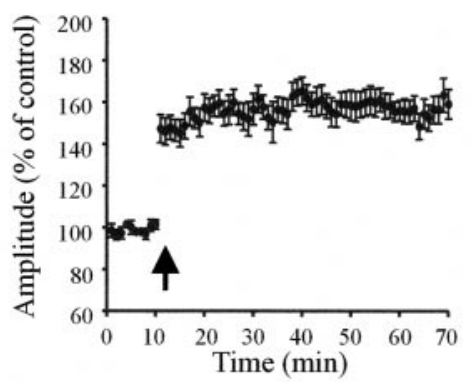

D

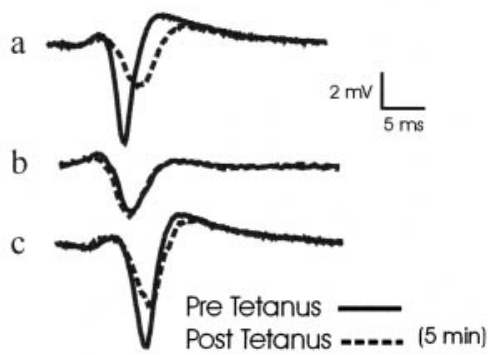

$\mathrm{D}$

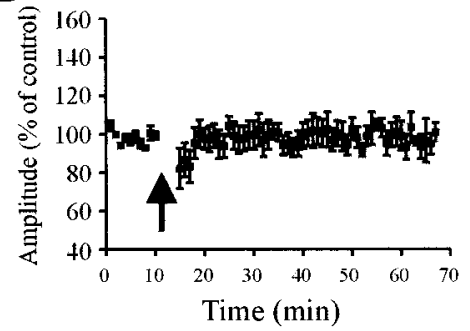

$\mathrm{E}$

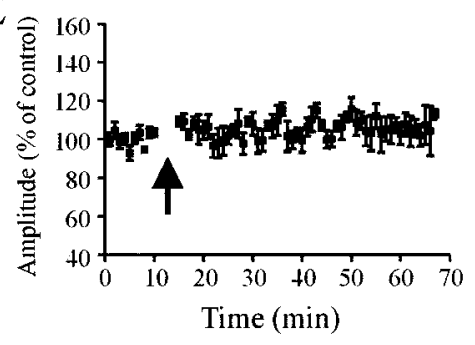

$\mathrm{F}$

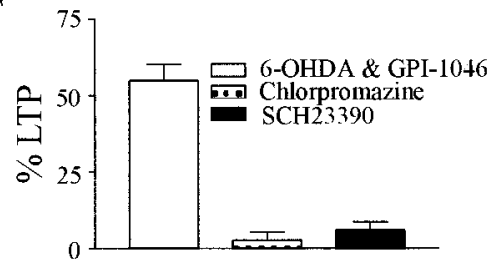

However the exact cascade is, as yet, unknown. The studies presented here also extend previous morphological findings (Steiner et al., 1997b). However, the results presented here and our previous ones (Steiner et al., 1997b) are in contrast to one other study in which only small changes in rotational activity or neuronal morphology were found in 6-OHDA-lesioned rats treated with GPI-1046 (Harper et al., 1999). In that study, the loss of striatal denervation and dopaminergic neurons in the substantia nigra was $\geq 95 \%$. Thus, if sprouting as a mechanism of func- tional recovery is important, perhaps the animals may have been lesioned too extensively. Thus, the beneficial effects of GPI-1046 may depend on there being sufficient substrate to induce significant striatal reinnervation and ultimately functional recovery.

In the hippocampus, in which LTP has been studied most extensively, its induction is primarily dependent on the activation of NMDA receptors (Connor et al., 1999). However, Frey et al. (1991) have demonstrated that blockade of D1 receptors with SCH23390 also decreased (not abolished) hippocampal LTP. 
SCH23390 was effective only when applied during, but not after, tetanic stimulation, indicating that endogenous DA was released by the tetanic stimulation. In addition, mice lacking D1 receptors do not express late-phase LTP in the hippocampus (Matthies et al., 1997). Thus, DA plays an important role in hippocampal LTP.

Corticostriatal LTP also is dependent on NMDA receptor activation; however, unlike in the hippocampus, it is completely dependent on dopamine action. Unilateral DA denervation by 6-OHDA injection into the substantia nigra blocks cortical striatal LTP in rats (Centonze et al., 1999b), providing direct evidence that the nigrostriatal dopamine system is required for the expression of corticostriatal LTP. The subtype specificity in the striatum seems to be the same as in the hippocampus, in which the D1 receptor activation augments LTP (Emmi et al., 1997). Because the hippocampus receives DA innervation from the ventral tegmental area and the substantia nigra (Gasbarri et al., 1994; Goldsmith and Joyce, 1994), it would be interesting to see whether hippocampal LTP is affected and/or restored by GPI1046 treatment as well. In any case, it appears that striatal LTP is dependent on DA, and DA is released by high-frequency stimulation. This conclusion is consistent with the findings of Ochi et al. (1995), who demonstrated that the release of $\mathrm{DA}$ by $\mathrm{KCl}$ was potentiated by high-frequency tetanic stimulation via an NMDA receptor-dependent pathway.

Given their similarities, it seems likely that both forms of LTP are dependent on the activation of cAMP-dependent PKA, which interacts with dopamine and the $32 \mathrm{kDa}$ cyclic $3^{\prime}, 5^{\prime}$ monophosphate-regulated phosphoprotein (Bach et al., 1999; Calabresi et al., 2000). However at the synaptic level, it is not clearly understood how the nigrostriatal dopaminergic system modulates the expression of corticostriatal LTP. One study has shown that the number of dendritic spines on the principal projection neurons in 6-OHDA-lesioned rats in the striatum was greatly decreased compared with normal animals; this decrease presumably results from the loss of nigrostriatal dopaminergic input. Accompanying this loss of the dendritic spines, the number of cortical terminals that terminate on the spines was greatly decreased (Ingham et al., 1998). Thus, it seems likely that the loss of LTP was attributable to a decreased number of cortical inputs that are normally recruited during a tetanic stimulation. Other possible ways in which the nigrostriatal DA system could regulate corticostriatal LTP may involve more complex neural circuits. Centonze et al. (1999a) has proposed that striatal LTP requires both principle projecting neurons and various interneurons in the striatum, which play a permissive role in the induction of and maintenance of LTP.

The concomitant loss of DA and LTP in the striatum provides a mechanism of synaptic plasticity, which may explain the underlying pathophysiology of motor rigidity or loss of automated movement, a classic hallmark of PD. A number of studies support the view that DA activity is important for inducing and maintaining synaptic plasticity. In goldfish reticulospinal neurons, direct application of DA to dendrites evoked potentiation of the synaptic response (Kumar and Faber, 1999). The D1 subtype plays a central role because mice lacking D2 but not D1-D5 receptors show LTP, whereas in the wild-type mice, long-term depression was seen (Calabresi et al., 1997). However, it has also been shown that rats lacking D1 receptors do not have significant motor deficits, and D1 agonists are only partially efficacious in treating PD. D2 receptors seem to be more important for the regulation of motor behaviors and are likely tonically activated by the continuous release of DA (Berke and Hyman, 2000). Nevertheless, this delineation of receptor subtype roles may also be influenced by the region of striatum in which the receptors are expressed because ventral regions may be more involved in addictive learned behaviors rather than in "pure" motor ones (Berke and Hyman, 2000). Here we show that DA is available for release; therefore, its re-expression in the striatum also should stimulate D2 receptors, and this may be more related to the loss of turning behavior than the LTP.

In conclusion, the medical benefits of immunophilin ligands seem to hold great potential for the treatment of PD and perhaps other neurodegenerative disorders. It is exciting to note that these results were obtained after a single course of treatment. We have yet to assess how long-term these changes may be. Other issues as to the source of the fibers will need to be addressed to determine the site of action of the drug as well. In any case, these data point to an important new avenue of hope for those who suffer from PD.

\section{REFERENCES}

Arbuthnott GW, Ingham CA, Wickens JR (2000) Dopamine and synaptic plasticity in the neostriatum. J Anat 196:587-596.

Bach ME, Barad M, Son H, Zhuo M, Lu YF, Shih R, Mansuy I, Hawkins RD, Kandel ER (1999) Age-related defects in spatial memory are correlated with defects in the late phase of hippocampal long-term potentiation in vitro and are attenuated by drugs that enhance the cAMP signaling pathway. Proc Natl Acad Sci USA 96:5280-5285.

Berke JD, Hyman SE (2000) Addiction, dopamine, and the molecular mechanisms of memory. Neuron 25:515-532.

Calabresi P, Pisani A, Mercuri NB, Bernardi G (1994) Post-receptor mechanisms underlying striatal long-term depression. J Neurosci 14:4871-4881.

Calabresi P, Saiardi A, Pisani A, Baik JH, Centonze D, Mercuri NB, Bernardi G, Borrelli E (1997) Abnormal synaptic plasticity in the striatum of mice lacking dopamine D2 receptors. J Neurosci 17:4536-4544.

Calabresi P, Gubellini P, Centonze D, Picconi B, Bernardi G, Chergui K, Svenningsson P, Fienberg AA, Greengard P (2000) Dopamine and cAMP-regulated phosphoprotein $32 \mathrm{kDa}$ controls both striatal longterm depression and long-term potentiation, opposing forms of synaptic plasticity. J Neurosci 20:8443-8451.

Centonze D, Gubellini P, Bernardi G, Calabresi P (1999a) Permissive role of interneurons in corticostriatal synaptic plasticity. Brain Res Brain Res Rev 31:1-5.

Centonze D, Gubellini P, Picconi B, Calabresi P, Giacomini P, Bernardi $\mathrm{G}$ (1999b) Unilateral dopamine denervation blocks corticostriatal LTP. J Neurophysiol 82:3575-3579.

Connor JA, Petrozzino J, Pozzo-Miller LD, Otani S (1999) Calcium signals in long-term potentiation and long-term depression. Can J Physiol Pharmacol 77:722-734.

Emmi A, Rajabi H, Stewart J (1997) Behavioral and neurochemical recovery from partial 6-hydroxydopamine lesions of the substantia nigra is blocked by daily treatment with D1/D5, but not D2, dopamine receptor antagonists. J Neurosci 17:3840-3846.

Frey U, Matthies H, Reymann KG, Matthies H (1991) The effect of dopaminergic D1 receptor blockade during tetanization on the expression of long-term potentiation in the rat CA1 region in vitro. Neurosci Lett 129:111-114.

Gasbarri A, Packard MG, Campana E, Pacitti C (1994) Anterograde and retrograde tracing of projections from the ventral tegmental area to the hippocampal formation in the rat. Brain Res Bull 33:445-452.

Goldsmith SK, Joyce JN (1994) Dopamine D2 receptor expression in hippocampus and parahippocampal cortex of rat, cat, and human in relation to tyrosine hydroxylase-immunoreactive fibers. Hippocampus 4:354-373.

Harper S, Bilsland J, Young L, Bristow L, Boyce S, Mason G, Rigby M, Hewson L, Smith D, O'Donnell R, O'Connor D, Hill RG, Evans D, Swain C, Williams B, Hefti F (1999) Analysis of the neurotrophic effects of GPI-1046 on neuron survival and regeneration in culture and in vivo. Neuroscience 88:257-267. 
Ingham CA, Hood SH, Taggart P, Arbuthnott GW (1998) Plasticity of synapses in the rat neostriatum after unilateral lesion of the nigrostriatal dopaminergic pathway. J Neurosci 18:4732-4743.

Kumar SS, Faber DS (1999) Plasticity of first-order sensory synapses: interactions between homosynaptic long-term potentiation and heterosynaptically evoked dopaminergic potentiation. J Neurosci 19:1620-1635.

Matthies H, Becker A, Schroeder H, Kraus J, Hollt V, Krug M (1997) Dopamine D1-deficient mutant mice do not express the late phase of hippocampal long-term potentiation. NeuroReport 8:3533-3535.

Ochi M, Inoue H, Koizumi S, Shibata S, Watanabe S (1995) Long-term enhancement of dopamine release by high frequency tetanic stimulation via a $N$-methyl-D-aspartate-receptor-mediated pathway in rat striatum. Neuroscience 66:29-36.

Steiner JP, Connolly MA, Valentine HL, Hamilton GS, Dawson TM,
Hester L, Snyder SH (1997a) Neurotrophic actions of nonimmunosuppressive analogues of immunosuppressive drugs FK506, rapamycin, and cyclosporin A. Nat Med 3:421-428.

Steiner JP, Hamilton GS, Ross DT, Valentine HL, Guo H, Connolly MA, Liang S, Ramsey C, Li JH, Huang W, Howorth P, Soni R, Fuller M, Sauer H, Nowotnik AC, Suzdak PD (1997b) Neurotrophic immunophilin ligands stimulate structural and functional recovery in neurodegenerative animal models. Proc Natl Acad Sci USA 94:2019-2024

Ungerstedt U (1971a) Postsynaptic supersensitivity after 6-hydroxydopamine induced degeneration of the nigro-striatal dopamine system. Acta Physiol Scand Suppl 367:69-93.

Ungerstedt U (1971b) Striatal dopamine release after amphetamine or nerve degeneration revealed by rotational behaviour. Acta Physiol Scand Suppl 367:49-68. 